\title{
Modeling and Simulation of CSMA/CA Slotted and Unslotted Mode in Zigbee Routing Schemes
}

\author{
Amritpal Kaur \\ M-Tech Scholar \\ Deptt of ECE \\ SBSSTC, Ferozepur, Punjab, \\ India
}

\author{
Jaswinder Kaur \\ Asst. Prof. \\ Deptt of ECE \\ SBSSTC, Ferozepur, Punjab, \\ India
}

\author{
Gurjeevan Singh \\ DIC-ECE \\ Deptt of ECE \\ SBSSTC (Polywing), Ferozepur, \\ Punjab, India
}

\begin{abstract}
Zigbee is basically a self organizing digital radio network. It is based on IEEE 802.15.4 standard used for Wireless Sensor Network. This research work is related to CSMA/CA slotted and unslotted mode on IEEE 802.15.4 based wireless sensor network using three routing schemes. The simulation model is done by using OPNET 14.5. Performances metrics like throughput, data traffic sent and received, packet dropped in the network are determined and analyzed. The results show that the star topology in beacon enable mode give better result than the non beacon mode and overall performance is superior then other topologies.
\end{abstract}

\section{Keywords}

WSN, CSMA/CA, Zigbee, Network Topologies, Opnet 14.5.

\section{INTRODUCTION}

Wireless sensor networks (WSN) are regularly used for realtime applications, such as environment examination, therapeutic care, and automobile traffic control. The sensor nodes have depict some source limits, which are not withstanding accurate under these circumstances. WSNs have to afford an unwavering coverage of the area of curiosity and also to get together rigorous time control activities [1]. Zigbee is a wireless personal area network based on IEEE 802.15.4 wireless protocol. Zigbee network defined first in 2004 and released in 2006. The second stack of the Zigbee network was defined as Zigbee 2006. It provides short distance communication with low complexity, low data rate and low power consumption. It is a two way technology which pointed to Wireless Sensor Network (WSN) [2]. Furthermore, it has several advantages such as self organization, smaller size of protocol stacks, and larger addressing space. Most commonly Zigbee also used in the medical field for patient monitoring or health and added together with self-care and self-management technologies can enhance their health outcomes [3]. The main aim of this technology is remote control and sensor applications, which is appropriate to operate in ruthless radio environments and isolated locations. IEEE 802.15.4 based WSN defines the physical and MAC layers. It use CSMA / CA mechanism and solves the problem of channel access [4]. The MAC layer of IEEE 802.15.4 standards operates in two modes, they are beacon enabled and non-beacon enabled mode. In beacon enabled mode, beacon messages are transmitted periodically for network organization and management. Beacon enabled are synchronized and allows the mode to operate on slotted Carrier Sense Multiple Access with Collision Avoidance (CSMA/CA) mechanism. In nonbeacon mode nodes are not synchronized, because periodic beacon transmissions are absent. Therefore, this mode supported to unslotted CSMA/CA mechanism [5]. The IEEE 802.15.4 uses a slotted CSMA/CA algorithm with Guaranteed
Time Slot (GTS) mechanism. Which support time-critical applications, GTS allocate at the network coordinator. A superframe is used to transmit the packets. Each superframe is divided into Contention Access Period (CAP) and a Contention Free Period (CFP). In CAP nodes compete along with each other to send packets, and in CFP nodes have GTSs to send packets without contention [6]. The superframe is send between two consecutive beacons and the time between these two beacons known as Beacon Interval (BI), the Superframe Duration (SD) is an active period. where a Base Superframe Duration is $15.36 \mathrm{~ms}$ for a data rate of $250 \mathrm{kbps}$ in the $2.4 \mathrm{GHz}$ frequency band, and $\mathrm{BO}$ (Beacon Order) and SO (Superframe Order) are two integers ranging from 0 to 14 [7]. In case of non beacon enable mode, the medium access control is provided by an unslotted CSMA/CA (Carrier Sense Multiple Access/ Collision Avoidance) mechanism using a random backoff time based on backoff period and backoff Exponent (BE). In this paper we analysis the result on the basis of different parameters for both of two beacon enable and non beacon enable mode over the different Zigbee routing schemes using mobile nodes.

\section{SYSTEM ARCHITECTURE}

Zigbee builds upon the physical layer and media access control defined in IEEE standard 802.15.4 for low-rate WPANs. The application layer is highest-level layer in the Zigbee specification. The layer allow effective interface to the user. A single node can support 240 applications, where number 0 is reserved for the Zigbee Device Object. An application converts input into digital data, and/or converts digital data into output. Several applications can be run over these nodes. The application support sub layer (APS) provides the services necessary for endpoints and the Zigbee Device Object (ZDO) perform data and management services to interface with the network layer. Some of the services like data transfer confirm, and responses are request provided by the APS to the application objects (end points). Zigbee devices object control and manage the application objects. The Zigbee device objects can perform function like representing node type of the device and also define its role in the network. The basic channel access mode is "carrier sense, multiple access/collision avoidance" (CSMA/CA). That is, the nodes talk in the same way that human's converse; they briefly check to see that no one is talking before they start, with three notable exceptions. Beacons are sent on a fixed timing schedule and do not use CSMA. Message acknowledgments also do not use CSMA. Finally, devices in beacon-enabled networks that have low latency real-time requirements may also use Guaranteed Time Slots (GTS), which by definition do not use CSMA [8]. The MAC sub layer needs to process data received by the physical layer in finite time duration. The inter frame space (IFS) defines the 
amount of time that separates the transmission of two consecutive frames. The IFS follows the acknowledgement frame during acknowledgment transmission, otherwise it follows the frame itself. The length of an IFS frame depends on the frame size. The transmission of short frames, which sizes are lower than a Max SIFS frame size $=18$ Bytes, is followed by short IFS (SIFS). The transmission of long frames, whose size are greater than a Max SIFS frame size, is followed by long IFS (LIFS) [9].The physical layer in IEEE 802.15.4 standard offers 27 channels, with a peak data-rate of 250Kbits/s. The MAC layer sorted nodes into network known as Personal Area Networks (PAN). A PAN is started by a router node (Coordinator), which assumes the role of parent. The parent and children nodes are combined to form a large PAN. Nodes within a PAN may be synchronized (beaconenabled mode) to communicate in a TDMA mode [10]. A Zigbee network implemented using three types of devices: 1) a coordinator; 2) multiple routers; and 3) multiple end devices. The coordinator format, maintain and perform all control functions in the network. A router is responsible for routing data between the end devices and the coordinator. An end device has to only receive the data and perform its specified function and its hardware requirements are minimized to manage cost. With the three types of devices, the Zigbee standard supports the following three network topologies: 1) star networks; 2) cluster-tree networks; and 3) mesh networks. The star topology consists of a coordinator and several end devices. In this topology, the end device communicates only with the coordinator. Any packet exchange between end devices must pass along the coordinator. The main advantages of star topology are its simplicity and predictable, energy saving. The drawbacks are limited scalability. A Tree topology consists of a Coordinator and this coordinator linked with its children, which are basically the set of routers and end devices. A Router also connected with its children like another routers and end devices. Mesh topology provides greater flexibility than other topologies and cover multiple paths for messages within the network. If a particular router fails, then Zigbee self-healing mechanism will recover the network. Mesh topology is highly reliable and robust. The limitation of this topology is lower end-to end performance [11]. In a Multihop mesh topology each neighbor can synchronize and send/receive data from any other neighbor, so in order to avoid collisions a device should not transmit beacons at the same time of any of node transmit beacon [12]. In a Zigbee beacon-enabled tree network, nodes' wake/sleep times must be fixed in the way that each router wakes up twice in each cycle to receive its children's packets and to transmit packets to its parent, respectively. The coordinator wakes up once to receive its children's packets. In a tree network, the beacons are announced by coordinator and routers. However, in a mesh network, beacons are not allowed to transmit over regular time period. Beacons are an important mechanism to support power management [13]. In star topology beacon is transmitted by a Zigbee coordinator.

\section{SIMULATION SCENARIO}

The simulation process is done on the basis of different scenarios using OPNET modeler 14.5. In these three scenarios using star, tree and mesh topologies are considered and each scenario has three mobile Zigbee coordinators. The network in each scenario contains equal number of mobile sensor nodes. The performance of these routing schemes evaluate using CSMA/CA slotted and unslotted mode under different network configuration.
Table 1: Simulation Parameters

\begin{tabular}{|c|c|}
\hline Network Scale & $500 \mathrm{~m} * 500 \mathrm{~m}$ \\
\hline Number Of Nodes & 85 \\
\hline Types of nodes & mobile \\
\hline Simulation Duration & $1200 \mathrm{~s}$ \\
\hline
\end{tabular}

Using Table 1, three scenarios has been designed in which the nodes are placed randomly anywhere in the network. For evaluation of the Zigbee network, the scenario describe as following below:

In first scenario, the tree routing schemes are implemented in a network with beacon enable or non beacon enable mode implemented one by one. Second scenarios design by star topology using both Beacon and Non Beacon enable mode. Third scenarios design by mesh topology using both Beacon and Non Beacon enable mode. Each network model consists of three ZC.

\section{RESULTS AND DISCUSSIONS}

On the basis of above assumptions following result have been observed:

\subsection{Throughput}

Throughput is the ratio of the total amount of data that a receiver receives from a sender to a time it takes for receiver to get the last packet. Throughput is the data quantity transmitted correctly starting from the source to the destination within a specified time (seconds). A low delay in the network translates into higher throughput. Throughput is quantified with varied factors including packet collisions, obstructions between nodes and the type of used topology. In the given figure 1, the throughput of star topology in beacon enable mode increases as simulation time increases and give highest throughput among all the topologies in the end. The transmissions in star topology is in the single depth and the coordinator first send beacons and then sleep in between the duration when beacon sent by it and further receive by any device. Where the star topology only consist of single coordinator and number of end devices so the communication between the end devices is only possible through coordinator. There will be the less obstruction in the network and coordinator only send data to the required one end device after sensing the channel (when channel is free), hence there will be the less collision.

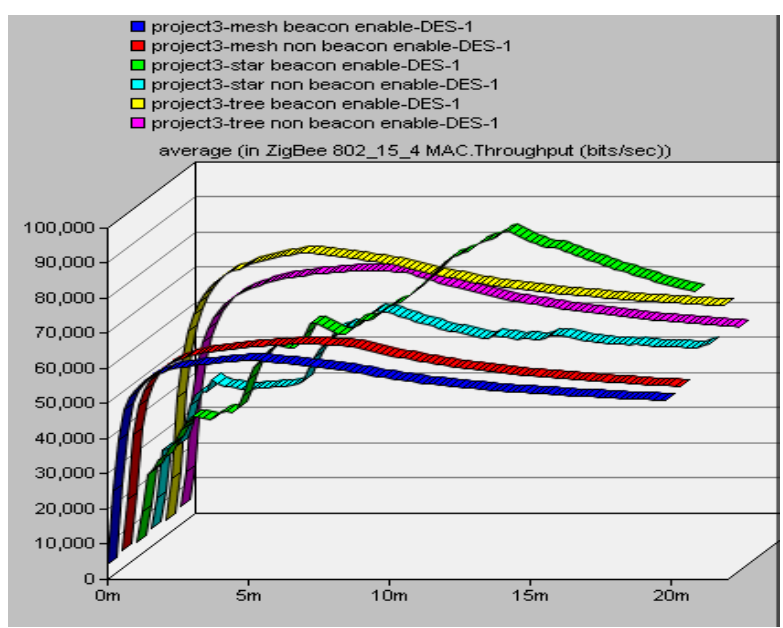

Fig 1: Throughput of Zigbee Topologies operating in Beacon Enable and Non Beacon Enable mode 


\subsection{Data Traffic Sent}

These statistics record the amount of data transmitted by the network interface onto the physical layer. When these statistics are reported in units of bits/second, the physical and the MAC header sizes are included in the computation of the total amount of traffic sent [15]. The maximum data traffic sent by star topology operated in beacon enable mode, as there is single hop transmission between the coordinator and end devices and due to multihop transmission minimum data traffic sent by mesh topology operated in non beacon enable mode as configured in figure 2 .

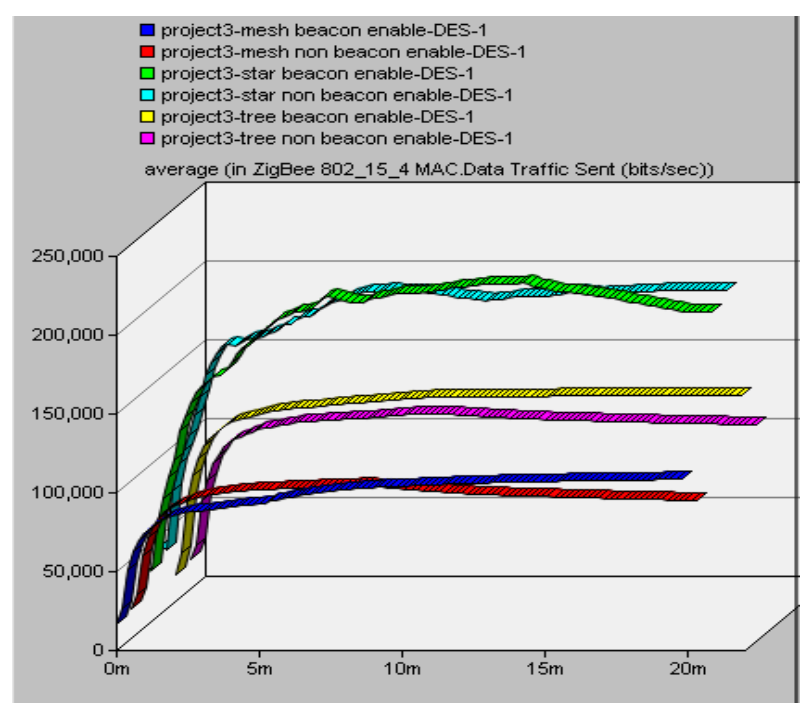

Fig 2: Data Traffic Sent by Zigbee Topologies operating in Beacon Enable and Non Beacon Enable mode

\subsection{Data Traffic Received}

These statistics record successfully received data traffic on this network interface from the physical layer. When these statistics are reported in units of bits/second, the physical and the MAC header sizes are included in the computation of the total amount of traffic received. These statistics record all the data received on the network interface regardless of the destination address. The result in figure 3, shows that maximum data traffic received by star topology operated in beacon enable mode and minimum data traffic received by mesh topology operated in beacon enable mode. But this data includes duplicate packets as the received data is more in number than sent data.

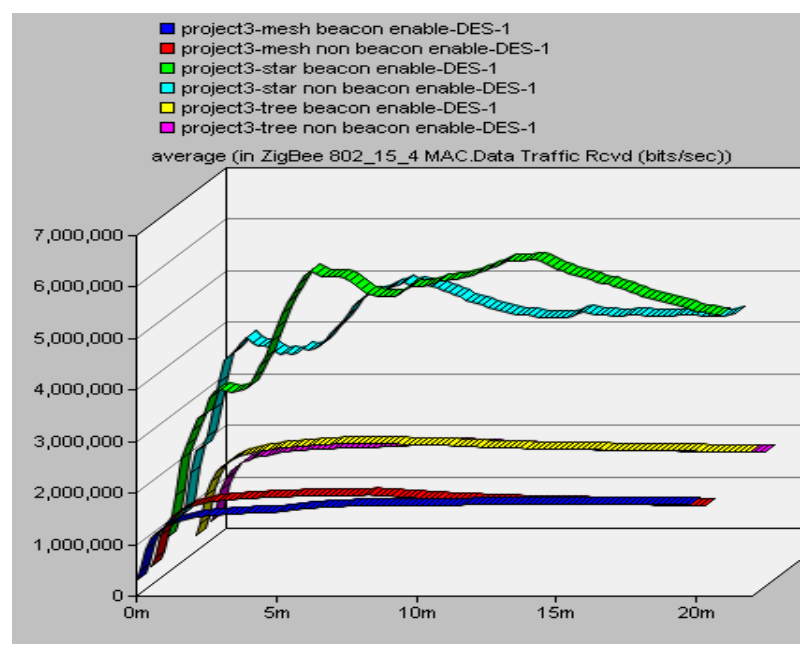

Fig 3. Data Traffic Received by Zigbee Topologies operating in Beacon Enable and Non Beacon Enable mode
Here in both cases of DTS and DTR the good response given by the star topology in beacon enable mode. Star topology work better in beacon enable mode as compared to non beacon mode because in non beacon mode coordinator without any beacon send the data either any end device is present in the network or not to receive the data. As in case of DTS star topology in both modes almost gives the similar response whereas in case of DTR, beacon enable mode gives maximum value due to the coordinator sent beacons for discovering the devices which has to be receive data from it. In case of mesh and tree topologies there will be multihop transmission so all the devices don't communicate directly with the coordinator.

\subsection{Packet Dropped}

This statistic show the packet not joined at the Network Layer. The figure 4 given below show that maximum packet dropped by tree topology, when operated in non beacon enable mode and star beacon has fewer packet dropped. These packets will be dropped at network layer while it will communicate to its highest layer either by its lower layer.

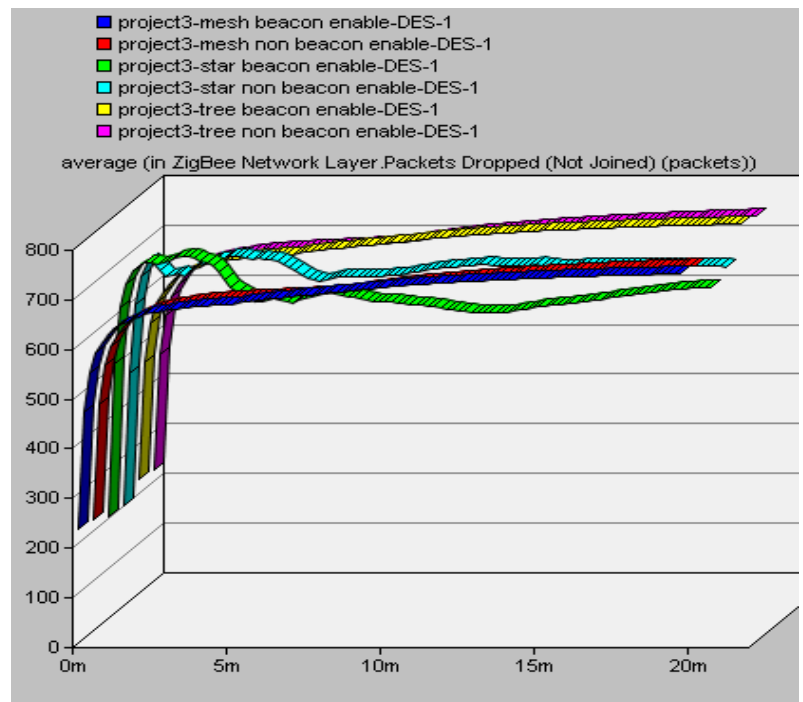

Fig 4. Packet Dropped by Zigbee Topologies operating in Beacon Enable and Non Beacon Enable mode

\section{CONCLUSION}

From the simulation results, it is observed that in case of throughput star topology in beacon enable mode increases as simulation time increases and give highest throughput among all the topologies in the end. The maximum data traffic received/sent by star topology operated in beacon enable mode and minimum data traffic received/sent by mesh topology operated in beacon enable mode. Maximum packet dropped by tree topology, when operated in non beacon enable mode and star beacon has fewer packets dropped. The overall results show that star topology with beacon enable mode performance is best than mesh and tree topology, while star topology in beacon enable mode also give good performance than non beacon mode.

\section{REFERENCES}

[1] Marco Gribaudo, Daniele Manini, Alessandro Nordio, "Transient Analysis of IEEE 802.15.4 Sensor Networks", IEEE Transaction On Wireless Communications, VOL.10, NO. 4, APRIL 2011. 
[2] Zavosh Abdollahzadeh Davani, Azizah Abdu Manaf, "A Survey on Key Management of ZigBee Network", The International Conference on E-Technologies and Business on the Web (EBW2013).

[3] Rozeha A. Rashid, Hamdan Sayuti, Nurul Mu'azzah Abdul Latiff, Norsheila Fisal, Mohd Adib Sarijari, Abdul Hadi Fikri Abdul Hamid, Rozaini Abd Rahim, "Simple Scheduling Scheme for Smart Home and Ambient Assisted Living", The Second International Conference on Informatics Engineering \& Information Science (ICIEIS2013) - Malaysia.

[4] Shi Longlonga, Qiu Chunlinga, Gao Penga, Jia Zhengsena, "The Research and Simulation of CSMA/CA Mechanism of Zigbee Protocol", 2012 International Workshop on Information and Electronics Engineering (IWIEE), Procedia Engineering 29 (2012) 3466 - 3471 Engineering 00(2011) 000-000.

[5] Surender.R, P. Samundiswary, "Performance Analysis of Node Mobility in Beacon and Non-Beacon enabled IEEE 802.15.4 based Wireless Sensor Network", IJCA (0975 - 8887), Volume 76- No.12, August 2013.

[6] Faiza Charfi, Mohamed Bouyahi, "Performance Evaluation Of Beacon Enabled IEEE 802.15.4 Under NS2",International Journal of Distributed and Parallel Systems (IJDPS) Vol.3, No.2, March 2012.

[7] Li-Hsing Yen, Yee Wei Law Marimuthu Palaniswami, "Risk-Aware Beacon Scheduling for Tree-Based Zigbee/IEEE 802.15.4 Wireless Networks" IEEE transactions on mobile computing, vol. 11 no. 4, April 2012.

[8] ZigBee - Wikipedia http://en.wikipedia.org/wiki/ZigBee.
[9] N. Boughanmi, YQ. Song, E. Rondeau, "Wireless Networked Control System using Zigbee/IEEE 802.15.4", 13th I Symposium on Information Control Problems in Manufacturing INCOM'2009, Moscou: Russian Federation (2009).

[10] S. Xu and R. Kumar, "Correct-by-Construction and Optimal Synthesis of Beacon-Enabled ZigbeNetwork", Automation Science and Engineering, IEEE Transactions on (Volume:10, Issue: 1).

[11] Yuan-Yao Shih, Wei-Ho Chung, Pi-Cheng, Hsiu, AiChun Pang, "A Mobility-Aware Node Deployment and Tree Construction Framework for Zigbee Wireless Networks",IEEE Transactions On Vehicular Technology, Vol. 62, No. 6, July 2013.

[12] Lalit Saraswat, Pankaj Singh Yadav, Rekha Rani, "Adaptability of IEEE 802.15.4(Zigbee) Protocol for Wireless sensor network", IJCSE, Vol. 02, No. 03, 2010, 554-559.

[13] Meng-Shiuan Pan, Yu-Chee Tseng, "Quick convergecast in Zigbee beacon-enabled tree-based wireless sensor networks", ELSEVIER, Computer Communications 31 (2008) 999-1011.

[14] Adarshpal S. Sethi,Vasil Y. Hnatyshin, "The Practical OPNET® User Guide for Computer Network Simulation", (C) 2013 by Taylor \& Francis Group, International Standard Book Number-13: 978-1-43981206-8.

[15] Shayma Wail Nourildean, “A Study of Zigbee Network Topologies for Wireless Sensor Network with One Coordinator and Multiple Coordinators", Tikrit Journal of Engineering Sciences/Vol.19/No.4/December 2012, $(65-81)$ 